\title{
Simulation and Modelling of the Solid Electrolyte Interphase with Varying Porosity
}

\author{
F. Single ${ }^{\mathrm{ab}}$, E. Karaca ${ }^{\mathrm{abc}}$, B. Horstmann ${ }^{\mathrm{ab}}$ and A. Latz ${ }^{\mathrm{abc}}$ \\ ${ }^{a}$ Institute of Engineering Thermodynamics, German Aerospace Center, 70569 Stuttgart, \\ Germany \\ ${ }^{\mathrm{b}}$ Helmholtz Institute Ulm for Electrochemical Energy Storage (HIU), 89081 Ulm, \\ Germany \\ c Institute of Electrochemistry, University of Ulm, 89081 Ulm, Germany
}

\begin{abstract}
We extend a popular approach in SEI modeling by refraining from using a single reaction interface. Instead we trace the time evolution of the SEI volume fraction in a spatially resolved way. In this study, we test how neutral lithium interstitial diffusion can explain the continuous growth of the SEI. We observe that transport of neutral lithium interstitials, as estimated by DFT calculation, is too slow to explain the growth of SEI layers with several tens of nanometer thickness. In future work, this spatially resolved transport and reaction framework will be used to study more realistic transport mechanisms.
\end{abstract}

\section{Introduction}

Continuous capacity fade during the lifetime of lithium ion batteries is mainly attributed to the growth of the solid electrolyte interphase (SEI). This layer forms on the negative electrode and consists of electrolyte reduction products. Active lithium that is consumed in this reduction reaction is no longer available for the primary battery function, leading to capacity fade. By preventing direct electrolyte/electrode contact, the SEI layer can suppress the electrolyte reduction reaction to a large degree. This means capacity fade and SEI growth still continue with a decreasing speed (1,2). The fundamental mechanisms behind this continuous SEI formation are still subject of active research.

Numerous models attempt to describe this effect (2-5). They typically assume that the formation rate is limited by a single transport mechanism that allows one reactant to cross the SEI layer. While most of these models predict the experimentally observed SEI thickness and capacity decay, they remain inconclusive with respect to the limiting transport process. We present and evaluate the results of a new model to describe the SEI growth. Our instead is based on two rate limiting processes and contains a spatiallyvarying porosity. This novel approach allows us to track the growth of the SEI morphology distribution.

We use this method to test a proposal by Shi et al (6). They calculated the concentration and the diffusion coefficient of neutral lithium interstitials in lithium carbonate and suggested that their transport might be the rate limiting mechanism that drives long term SEI growth. 


\section{Modeling Approach}

We simulate the evolution of the porous SEI layer in one dimension perpendicular to the electrode surface. This leads to the assumption that the SEI is homogeneous parallel to the electrode surface, i.e. impurities are neglected. For simplicity we assume that the SEI consists of a single component, formed by the reduction of solvent. The electrolyte is assumed to be a binary mixture of solvent and inert cosolvent, treated as an incompressible fluid.

Lithium interstitials diffuse through the SEI phase making the lithium interstitial diffusion coefficient a parameter of our model. Solvent is transported in the electrolyte phase by diffusion and convection (volume conservation during the formation process drives the convection current). The transport process in each phase is schematically depicted in Fig. 1.

The effective diffusion coefficients depend on the local porosity of the SEI layer. This is modeled with the Bruggeman ansatz. While the Bruggeman coefficient in the SEI phase is chosen to be the standard one, the Bruggeman coefficient in the electrolyte phase is a parameter of our model.

The reaction kinetics is modeled with a Butler-Volmer rate, affected by the local activities of lithium interstitials and active solvent.

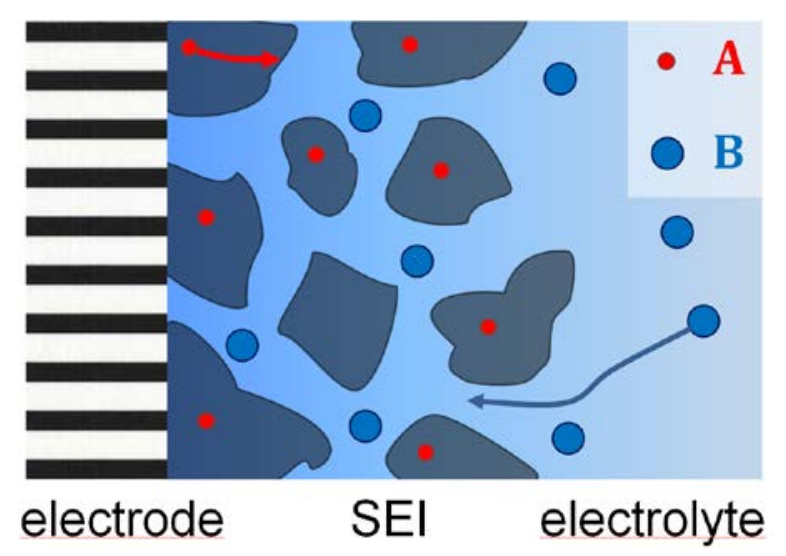

Figure 1. Modeling scheme. The SEI is a porous layer that grows on the electrode surface. Inside the SEI phase neutral lithium interstitials (A) can diffuse and eventually react with the main solvent (B) that is transported in the electrolyte phase. They can react everywhere within the simulation domain to form new SEI.

\section{$\underline{\text { Results and Conclusion }}$}

Our simulations show that the SEI thickness grows with the square root of time. Thus, the model agrees with its predecessors (2-5) as well as with experiments $(1,7)$. SEI growth is very slow when using the results from (6) for the lithium interstitial concentration at the electrode and the lithium interstitial diffusion coefficient. The growth rate can be accelerated to experimentally observed levels by increasing one or both of these parameters.

We observe a finite porosity in the SEI. A more detailed description of this simulation method and a more detailed analysis of the results will be presented in future work. 


\section{References}

1. Smith, a. J., Burns, J. Electrochem. Soc., 158, A447 (2011)

2. M. Pinson, M. Bazant, J. Electrochem. Soc., 160(2), A243-A250 (2012)

3. H. Ploehn, P. Ramadass, J. Electrochem. Soc., 151(3), A456 (2004)

4. M. Safari, M. Morcrette, J. Electrochem. Soc., 156(3), A145 (2009)

5. J. Christensen, J. Newman, J. Electrochem. Soc., 151(11), A1977 (2004)

6. S. Shi, P. Lu, J. American Chemical Soc., 134(37), 15476-15487 (2012)

7. P. Liu, J. Wang, J. Electrochem. Soc., 157, A499 (2010) 